\title{
Frontières
}

\section{Les médicaments antirétroviraux et le VIH/sida}

\section{Entre espoir et scepticisme}

\section{Stéphanie Argentier, Mylène Fernet, Joseph J. Lévy, Robert Bastien, René Fernet, Germain Trottier, Johanne Samson, Normand Lapointe, Joanne Otis, Marlène Rateau, Marie Harerimana, Antoine Bourdages et Marc Boucher}

Volume 16, numéro 1, automne 2003

Remède ou poison?

URI : https://id.erudit.org/iderudit/1073760ar

DOI : https://doi.org/10.7202/1073760ar

Aller au sommaire du numéro

Éditeur(s)

Université du Québec à Montréal

ISSN

1180-3479 (imprimé)

1916-0976 (numérique)

Découvrir la revue

Citer cet article

Argentier, S., Fernet, M., Lévy, J. J., Bastien, R., Fernet, R., Trottier, G., Samson, J., Lapointe, N., Otis, J., Rateau, M., Harerimana, M., Bourdages, A. \& Boucher, M. (2003). Les médicaments antirétroviraux et le VIH/sida : entre espoir et scepticisme. Frontières, 16(1), 44-50. https://doi.org/10.7202/1073760ar
Résumé de l'article

Les traitements médicaux pour lutter contre le VIH/sida ont été limités pendant la plus grande partie de l'histoire de l'épidémie marquée par de nombreux décès. Depuis les années 1990, la mise au point de nouveaux médicaments antirétroviraux, sans constituer une cure, ont contribué chez plusieurs à l'amélioration de l'état de santé. Pour d'autres personnes vivant avec le $\mathrm{VIH} /$ sida, les effets indésirables des médicaments ont remis en question l'intérêt de ce traitement. Cet article explore, à partir de témoignages de personnes, hommes et femmes, vivant avec le VIH/sida à Montréal, les représentations contradictoires, oscillant entre scepticisme et espoir, entourant l’usage de ces médicaments. 


\section{Résumé}

Les traitements médicaux pour lutter contre le VIH/sida ont été limités pendant la plus grande partie de l'histoire de l'épidémie marquée par de nombreux décès. Depuis les années 1990, la mise au point de nouveaux médicaments antirétroviraux, sans constituer une cure, ont contribué chez plusieurs à l'amélioration de l'état de santé. Pour d'autres personnes vivant avec le $\mathrm{VIH} /$ sida, les effets indésirables des médicaments ont remis en question l'intérêt de ce traitement. Cet article explore, à partir de témoignages de personnes, hommes et femmes, vivant avec le VIH / sida à Montréal, les représentations contradictoires, oscillant entre scepticisme et espoir, entourant l'usage de ces médicaments.

Mots clés: VIH/sida - traitements entrevues.

\section{Abstract \\ The medical treatments to fight HIVIAIDS were limited during most of the history of the epidemic, marked by many deaths. Since the 1990's, the development of a number of anti-retrovirals, while not constituting a cure, has contributed to better health for many. For others living with HIVIAIDS, the side-effects of these medications have put these treatments into question. This article explores, from interviews of men and women living with HIVIAIDS in Montreal, the contradictory representations, oscillating between scep- ticism and hope, around the use of these medications.}

Keywords: HIV I AIDS - treatmentinterview.

\section{Les médicaments antirétroviraux et le VIH / sida Entre espoir et scepticisme}

\author{
Stéphanie Argentier ${ }^{1}$, Mylène Fernet ${ }^{2}$, \\ Joseph J. Lévy ${ }^{2}$, Robert Bastien ${ }^{3}$, René Fernet ${ }^{4}$, \\ Germain Trottier ${ }^{5}$, Johanne Samson ${ }^{6}$, \\ Normand Lapointe ${ }^{7}$, Joanne Otis ${ }^{2}$, \\ Marlène Rateau ${ }^{8}$, Marie Harerimana ${ }^{9}$, \\ Antoine Bourdages ${ }^{10}$, Marc Boucher ${ }^{7}$.
}

À partir du XVIII ${ }^{\mathrm{e}}$ siècle, l'émergence des sciences biomédicales et pharmacologiques (Chast, 1995) a contribué à une compréhension de plus en plus fine du fonctionnement de l'organisme et des effets des substances chimiques, naturelles ou synthétiques, sur le traitement des maladies et l'amélioration de la santé physique ou mentale.

Ces progrès s'amplifient au XIX ${ }^{\mathrm{e}}$ siècle alors que les médicaments modernes produits industriellement se généralisent, donnant lieu à des classes de médicaments de plus en plus efficaces (sulfamides, antihistaminiques, neuroleptiques, antibiotiques). Plus récemment sont apparus des biotechnologies de plus en plus raffinées qui révolutionnent le traitement d'un large registre de maladies, depuis les troubles mentaux jusqu'aux dysfonctions sexuelles, non sans se heurter cependant, dans ce parcours, à des obstacles importants, comme le montrent les tentatives de traitement du VIH / sida, suivant l'apparition de cette épidémie au début des années 1980.

Événement majeur au plan socioculturel, l'épidémie de sida allait profondément déstabiliser les sociétés modernes en remettant en question certaines de ses valeurs sociales et éthiques et, en particulier, le sentiment de toute-puissance de l'establishment biomédical qui se voyait confronté à un virus dont les effets ravageurs pouvaient aller jusqu'à entraîner une mort rapide et ce, malgré la mise en marché en 1987 du premier nucléoside, l'AZT (zidovudine). Celui-ci fut suivi dans les années suivantes par d'autres médicaments du même type comme la didanosine (DDI), la zalcitabine (DDC), la lamivudine (3TC) et la stavudine (D4T). Ces substances ont été ainsi utilisées en monothérapie, puis, à cause de la résistance croissante du virus, à des bithérapies combinant l'AZT aux autres médicaments.

Cette situation s'est significativement modifiée à partir de 1996, lorsque, à la Conférence internationale sur le sida tenue à Vancouver, fut annoncée la mise au point d'une nouvelle classe de médicaments. Les inhibiteurs de la protéase (saquinavir, indinavir, ritonavir) ont constitué ainsi un point tournant dans les perspectives de traitement. Ainsi grâce aux HAART (highly active anti-retroviral treatment), des trithérapies et, dans certains cas, des quadrithérapies ont succédé aux bithérapies et elles ont consisté soit à combiner deux nucléosides à un inhibiteur de la protéase, soit à combiner un ou deux nucléosides et deux inhibiteurs de la protéase.

En 1998, enfin, est apparue une nouvelle famille de produits, les inhibiteurs non 
nucléosidiques de la transcriptase inverse, plus communément appelés non-nucluéosides (effavirenz, delavirdine, névirapine), qui ont étendu la panoplie des traitements disponibles et de plus en plus répandus. Ils ont ainsi contribué à améliorer l'état de santé général en réduisant la charge virale, non sans provoquer cependant des effets indésirables souvent importants qui allaient atténuer l'optimisme initial qui avait suivi leur mise au point et leur disponibilité.

Dans cette perspective, nous explorerons d'abord comment ces nouvelles thérapies ont changé les représentations de l'épidémie et le rapport à la mort à partir des recherches menées au plan sociologique et psychosocial. Dans un second temps, nous verrons comment la thérapie antirétrovirale est accueillie par les patients et comment elle transforme leur rapport à la mort et à la vie. Pour ce faire, nous nous baserons sur ce qui intervient sur la perception et les représentations des médicaments chez les personnes vivant avec le VIH (PVVIH) et leurs répercussions sur les questions existentielles rattachées à la maladie, la vie et la mort. Pour cerner ces dimensions, nous nous baserons sur les données de deux recherches qualitatives menées à Montréal auprès de PVVIH. La première étude portait sur un ensemble de 26 hommes ayant des relations avec d'autres hommes. Québécois francophones, leur âge se situait entre 21 et 54 ans. Parmi ceux-ci, 21 étaient sous traitement antirétroviral au moment de l'entrevue (Lévy et al., 2001). La seconde étude (Bastien et al., 2003), en cours, porte sur des femmes infectées et sous traitement (Québécoises d'origine, Antillaises et Africaines). Les données citées ici proviennent des entretiens avec huit femmes hétérosexuelles, d'origine africaine, âgées de 29 à 43 ans qui seront présentées. Parmi celles-ci, six étaient asymptomatiques, et cinq sous traitement antirétroviral au moment de l'entrevue.

Tous les patients ayant participé à ces recherches étaient suivis par un médecin au moment de l'entrevue. La recherche portait sur la trajectoire des répondants avec le VIH / sida, et la trajectoire avec la médication (sphères sociale, interpersonnelle et intime). L'analyse des données s'est effectuée à partir de la codification des entrevues et l'extraction de thématiques se rapportant aux impacts de la maladie et à l'effet des médicaments.

\section{RÉPERCUSSIONS DES TRAITEMENTS ANTIRÉTROVIRAUX SUR LES REPRÉSENTATIONS DE LA MALADIE}

Pendant les premières années de l'épidémie, le diagnostic d'infection par le VIH était associé généralement à une mort rapide à la suite de l'effondrement du sys- tème immunitaire des patients. Il y avait apparition de lésions, un amaigrissement notable, l'apparition de maladies pulmonaires inhabituelles et d'infections opportunistes graves. Les textes biomédicaux, journalistiques et littéraires, en traitant de la question des risques de contamination, $\mathrm{du}$ suicide et des soins palliatifs mettaient au cœur de leur discours la dimension thanatologique de l'épidémie du VIH/sida, avant tout perçue à travers sa spécificité fatale, foudroyante et transmissible. Cette représentation de la mort inscrivait alors l'aspect psychologique du deuil dans un processus général connu et bien documenté. En effet, les modèles de crises proposés par Freud, en 1917, ceux de transition popularisés par Élizabeth Kubler-Ross (1977), et les modèles contextuels, initiés par Lofland (1985), décrivent un mouvement similaire (Séguin et Fréchette 1995). Le deuil s'enclenche par une période de choc, débouchant sur des épisodes de désorganisation qui sont ralentis par un mouvement de colère, de résistance à la réalité de la mort. S'ensuit tôt ou tard une impasse dépressive, noyau d'une réorganisation intérieure incontournable face au processus du mourir.

Cette épidémie venait, dans les pays occidentaux, décimer surtout des hommes âgés de 25 à 44 ans chez qui le VIH / sida constituait alors la première cause de décès. Cet élément accentuait l'intensité du choc et de la résistance face à la mort. Ces coupes sombres affectaient en particulier les réseaux homosexuels confrontés à des deuils fréquents et successifs, transformant ainsi le rapport à la mort qui, d'invisible et privée, devenait de plus en plus publique et à la fois individualisée et collective. Devant ces décès nombreux, de nouvelles formes de mémorialisation (courtepointes, archives, décharge de ballons avec le nom des personnes décédées, allumage de bougies, installations architecturales commémoratives, journée mondiale du sida) ont été élaborées (Lévy et Otis, 2000). Ces rituels, en plus de briser le caractère de ghetto du milieu de vie des sidéens, tendent à démontrer cette perpétuelle quête d'expressions spirituelles, de type communautaire, entourant le deuil et la mort.

Dans le champ de la création littéraire et artistique, «la maladie ouvrirait donc non seulement à la conscience de la mortalité partagée mais autoriserait le récit de cette mortalité. Et l'écrivain se retrouve à témoigner non seulement pour lui-même mais aussi pour la communauté des sidéens, disparus ou encore vivants » (Nouss, 1997, p. 502). La médecine n'étant pas encore en mesure de proposer un traitement efficace, le rapport à la médication ne constituait pas un thème privilégié dans les débats concernant le VIH / sida, sinon pour dénoncer la lenteur des recherches. Démuni de moyens médicaux efficaces, le médecin s'occupait alors de la personne du mourant et de sa souffrance.

L'approche du traitement relevait en grande partie d'une perspective sociopsychologique où il s'agissait « d'étayer la qualité de (sur)vie des malades et d'actualiser la force du moi dans les limites du temps qui reste, [et doit] tôt ou tard s'ajuster aux particularités de la phase terminale qui fait davantage appel à l'accompagnement » (de Montigny 1997, p. 413). Par la suite, avec le développement des premières techniques thérapeutiques de prise en charge de l'infection par le $\mathrm{VIH} /$ sida qui a permis de prolonger la vie des personnes atteintes, ces dernières connurent un regain d'espoir, un mieux-être psychologique. Toutefois, l'enthousiasme ne fut que de courte durée. En effet, on assistait à une transition entre un temps de vie circonscrit à un autre, imprévisible. Dans la première situation, certains s'attardaient à préparer leur détachement et d'autres à exacerber leur désir de vivre. Dans la seconde situation, le contexte d'incertitude, occasionné par le temps à vivre avec le VIH qui devenait de moins en moins prévisible, intensifiait souvent l'angoisse de composer avec l'inconnu (de Montigny 1997). Cette incertitude sabote le processus du deuil. Le malade devient ambivalent entre se préparer à mourir dans un temps imprévisible ou à vivre avec une qualité de vie autre. Ainsi, dans le processus de deuil, celui qui est en période de résistance est appelé à nourrir sa résistance et à bloquer ses processus d'adaptation. L'autre, qui est en processus de réorganisation face au mourir, devient perplexe et démuni. Il peut regretter la manière dont il a disposé de ses avoirs pour finir sa vie dans un temps déterminé. Quant aux proches du mourant, doivent-ils l'encourager à s'engager dans une lutte à finir contre la mort ou l'accompagner pendant l'état de siège où la maladie le maintient en garde à vue ? En effet, les proches ont besoin eux aussi de comprendre les particularités des deuils distinctifs, étant eux aussi secoués par l'angoisse de composer avec l'inconnu (Fernet 1999).

Cette situation se transforme de façon radicale avec l'apparition des multithérapies en 1996. Celles-ci étaient alors célébrées comme une percée significative dans la lutte contre l'épidémie, ce que les rapports épidémiologiques européens et américains devaient confirmer. Ceux-ci notaient, avec l'usage des médicaments, plusieurs tendances : un allongement significatif de la période allant de l'infection par le VIH au développement du sida, puis au décès des personnes atteintes (Detels et al., 1998), ainsi qu'une chute significative dans le nombre de décès dus au VIH/sida, 
même si ces répercussions variaient en fonction des groupes de transmission et la disponibilité. Le Canada rapportait aussi une réduction (Santé Canada, 2003).

Cette prolongation significative de l'espérance de vie des PVVIH a tout transformé. Les progrès pharmaceutiques ont subitement changé la perception de l'épidémie, entraînant une forme de normalisation paradoxale du sida (Setbon, 2000). Le sida perd son statut d'exception pour se rapprocher davantage des caractéristiques d'une maladie chronique. En effet, l'horizon de la mort s'éloigne, atténuant ainsi la liaison sida-mort. Le processus du deuil de sa vie paraît rompu. Le deuil de sa qualité de vie devient le nouvel enjeu.

La médicalisation de la prise en charge du VIH / sida déplace les préoccupations sociopsychologiques au second plan. L'attention se porte sur la surveillance du suivi du traitement, sur l'évaluation des bilans biomédicaux et sur l'efficacité des thérapies, souvent aux dépens d'une écoute adéquate des enjeux associés chez plusieurs patients aux problèmes d'observance (respect des prescriptions, des consignes de traitement) et d'adhésion au traitement (capacité du patient à faire sien le traitement ; Delfraissy et al., 1999), ainsi qu'aux effets indésirables des médicaments chez les patients. Le médecin a retrouvé son rôle de compétence et le patient perd souvent la qualité de l'attention portée à sa personne et à ses souffrances.

\section{LES RÉPERCUSSIONS BIOMÉDICALES \\ DES ANTIRÉTROVIRAUX}

\section{SUR LE QUOTIDIEN DES PATIENTS}

L'observance rigoureuse des traitements, essentielle pour éviter le développement des résistances aux médicaments anti-VIH, se heurte non seulement à de nombreuses difficultés relevant des contraintes liées aux routines thérapeutiques, mais aussi aux conséquences associées à leur révélation à l'entourage familial et social. Les médicaments nécessitent en effet la mise en place d'une adaptation de la vie quotidienne au schéma thérapeutique, en fonction des traitements et doses. Même si, dans certains cas, les protocoles se sont simplifiés, il demeure souvent difficile d'assurer une prise régulière, en particulier lorsque les médicaments suscitent des réactions d'aversion liées à leur nombre, à leur goût et à leurs effets.

Plusieurs stratégies peuvent être ainsi adoptées pour assurer le rappel des prises (pilulier, alarmes) ou repenser les horaires en ce qui a trait au sommeil ou aux repas. Le traitement qui vient scander le temps vécu par le patient avec lourdeur et régula- rité, et ce pour une durée à long terme, renvoie sans cesse ce dernier à son statut d'individu malade ou susceptible de le devenir.

La question de la visibilité des traitements peut aussi interférer avec le suivi thérapeutique. Comme l'infection par le VIH est un aspect que l'on préfère garder caché ou partager avec des personnes choisies, les médicaments sont souvent considérés comme un révélateur, un signe extérieur tangible de la maladie dont il faut gérer la présence. Le fait de pouvoir être identifié comme PVVIH est donc un souci majeur à cause de l'isolement, de la stigmatisation ou de la discrimination qui pourraient découler de la divulgation du statut infectieux et médical, avec ses conséquences incertaines sur les relations sociales, familiales et intimes.

Le recours à des modalités d'occultation du traitement est donc quelquefois privilégié (éviter de prendre les médicaments en public ou en présence de certaines personnes, assurer la prise la plus discrète possible, cacher les médicaments, changer le contenant ou l'étiquette, etc.), ce qui rend d'autant plus difficile son intégration à la vie quotidienne, en particulier dans le cadre des relations intimes. Malgré les progrès médico-pharmaceutiques, les préoccupations face à la révélation de l'infection par le VIH restent donc prégnantes, ce qui suggère que le VIH / sida continue d'avoir un statut social d'exception.

Les effets secondaires indésirables, d'intensité variable en durée, en fréquence et en gravité selon les patients, et qui apparaissent à la suite de la médication viennent renforcer les perceptions que les médicaments ne contribuent pas toujours à un mieux-être. Ces effets secondaires s'expriment sous la forme de malaises affectant les fonctions corporelles plus ou moins inconfortables (par exemple, maux de tête, nausées, vomissements, éruptions cutanées, fatigue, douleurs ou malaises abdominaux, diarrhées, problèmes digestifs, altération du goût ou de l'odorat) ou, au contraire, entraînent des problèmes de santé à part entière (par exemple anormalités métaboliques, hyperlipidémie, niveaux de glucose élevés, lipodystrophie et / ou lipoatrophie ; dysfonctions sexuelles, insomnie, anxiété et dépression), nécessitant le recours à d'autres médicaments ou même, dans les cas les plus graves, à une hospitalisation.

La lipodystrophie et / ou la lipoatrophie, qui intervient sur le corps (émaciation du visage, des fesses et des membres ou accumulation d'une masse graisseuse au niveau de la taille) constitue l'une des manifestations les plus lourdes des effets des multithérapies. Au plan personnel, elle affecte l'apparence corporelle et l'image de soi, ce qui peut entraîner une baisse de l'estime de soi et de son potentiel de séduction et d'attraction, des éléments importants dans le milieu gai où certaines interactions s'inscrivent dans une dynamique très sexualisée. Pour les femmes d'orientation hétérosexuelle rencontrées, la dissimulation des parties du corps par l'agencement des vêtements est une option possible mais le visage restant

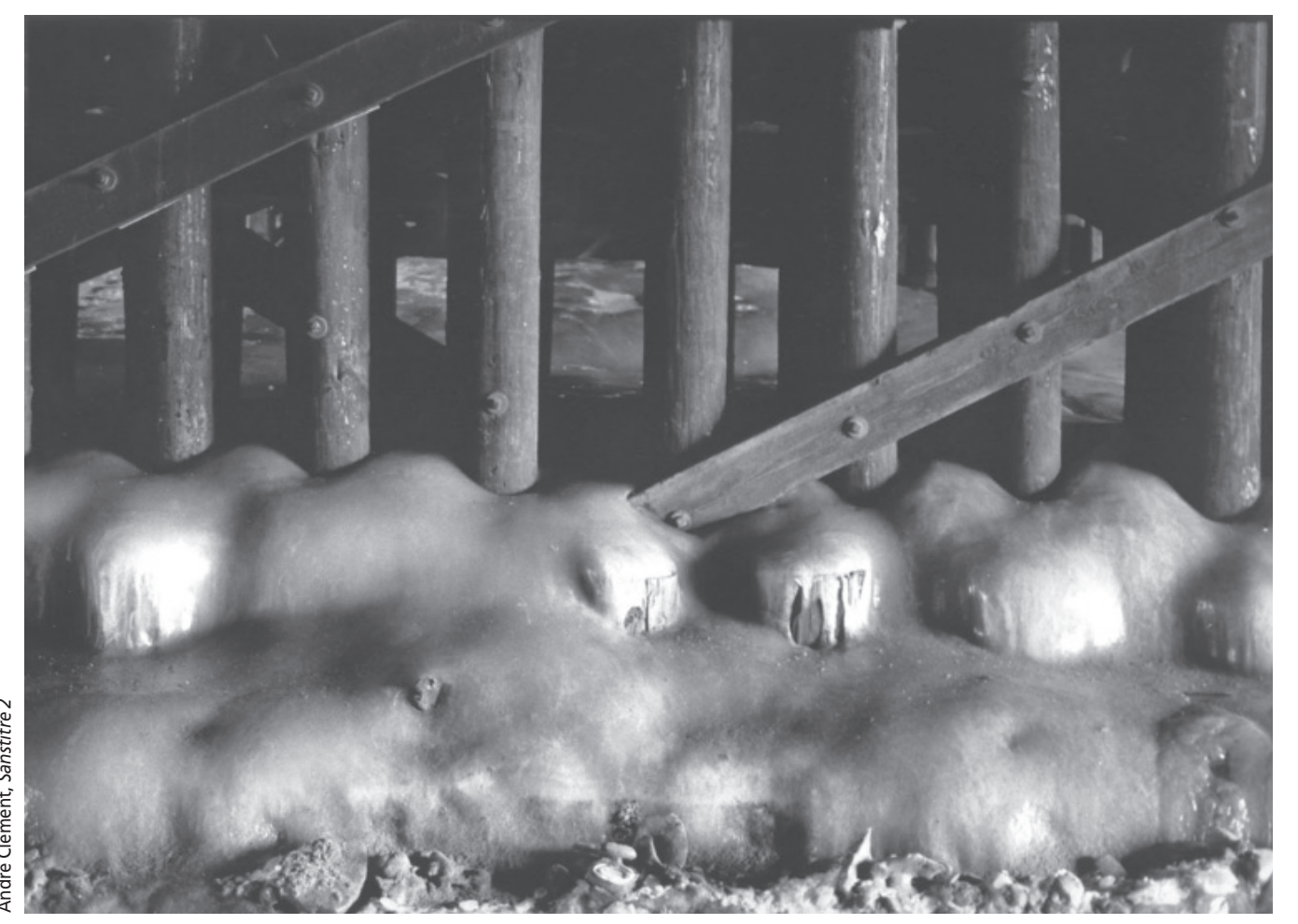


à découvert, son état d'émaciation devient un révélateur du statut infectieux que l'on tient pourtant à cacher.

Face à ces contraintes et ces effets, comment les patients sous traitement perçoivent-ils les médicaments ? Comment se les représentent-ils? C'est ce que nous verrons à présent à partir des données d'entrevues effectuées auprès de PVVIH homosexuels et de femmes d'orientation hétérosexuelle et d'origine africaine vivant à Montréal.

\section{PERCEPTIONS ET REPRÉSENTATIONS DES MÉDICAMENTS ANTIRÉTROVIRAUX}

L'information sur les médicaments provenant des intervenants biomédicaux ou d'autres sources (entourage, médias, organismes communautaires, etc.) semble différer entre les deux populations. Parmi les hommes, une grande proportion paraît très bien documentée et informée sur les diverses possibilités de prise en charge de la maladie et sur les effets indésirables des médicaments, ce qui semble entraîner une plus grande implication de leur part dans le processus thérapeutique et les prises de décisions qui lui sont associées. Les femmes ont tendance à accorder une plus grande confiance aux traitements pharmacologiques prescrits par leur médecin, même s'il semble qu'elles disposent de connaissances moindres concernant la maladie, les médications et les effets secondaires des traitements. Cependant, l'expérience associée aux traitements donne naissance à des constructions diverses dans lesquelles on peut opposer une vision positive des médicaments à une perspective plus ambivalente sinon franchement négative.

\section{LES MULTITHÉRAPIES :}

\section{SOURCE DE VIE ET D'ESPOIR}

Pour la majorité des personnes rencontrées en entrevue, l'accès aux multithérapies a constitué une formidable source d'espoir et de bien-être. Rejoignant la notion de revival (Brashers et al., 1999) liée à l'impact des nouvelles thérapies chez des patients ayant connu l'assaut des infections opportunistes, plusieurs parlent d'un renouveau, d'une seconde vie:

Les médicaments, ça m'a donné l'espoir, je dois dire que ça m'a fait revivre» (homme) ;

Je pense que ça été quelque chose d'extraordinaire ces médicaments bien qu'ils soient toxiques, ça donne de l'espoir (femme).

Si je continue les médicaments, ça me donne quand même l'espoir que je vais combattre ce virus. Il va peut-être être stabilisé, il ne va pas attaquer d'autres parties, d'autres cellules. Il va rester là et puis moi aussi je vais vivre. Tant que je peux les avoir, je les prendrai (femme).

J'ai toujours pensé que ça me fait du bien, je vais toujours continuer à prendre mes médicaments, et puis le médecin m'a dit de continuer à les prendre, parce c'est vraiment bien pour nous, ça me donne de la force, de l'énergie et tout et tout. Il ne faut pas arrêter donc, c'est sûr que je vais continuer à les prendre, sans problème (femme).

Rempart significatif contre la mort, qu'ils retardent, et contre les symptômes du sida, qu'ils atténuent ou font disparaître, ces produits pharmaceutiques, considérés comme des pilules miracles ou des panacées et porteuses de vie par les PVVIH, véhiculent l'espérance d'une guérison complète, ou tout au moins d'un contrôle de l'occurrence des symptômes du sida ou une rémission significative attribuée au combat du médicament contre le virus :

Si j'arrête les médicaments, je vais encore une fois développer le virus. Le virus va se développer dans mon corps, autant continuer à avoir ces effets indésirables. Je vais lutter, je vais essayer de vivre avec, mais je préfère quand même que le virus soit combattu, parce que mes CD4 maintenant c'est entre 50 et $0 \ldots$ c'est indétectable depuis plus de cinq mois (femme).

C'est sûr qu'ils font bien leur travail dans le corps, c'est ça que je sais. Ils doivent combattre le virus (femme).

Parmi les PVVIH homosexuels qui avaient déjà ressenti les premières conséquences du VIH avec l'apparition d'infections opportunistes, le traitement a souvent permis de stabiliser la progression de l'infection, et même dans certains cas de la renverser. Ainsi, plusieurs ont vu leur état de santé s'améliorer. Chez ceux qui étaient encore asymptomatiques au moment où les multithérapies sont apparues, le traitement a généralement réussi à maintenir l'infection par le VIH à son état latent. Dans les deux cas, de voir la charge virale ramenée à un seuil indétectable ou l'immunité se rétablir a généré une perception positive face aux médicaments. De ce fait, le rapport à la mort s'est estompé, en particulier parmi ceux qui ont une séroconversion récente, après que le choc de l'infection par le VIH ait été absorbé ou encore quand, une fois le suivi et le traitement entrepris, les tests confirment que le virus est dormant. Pour les autres, le rapport à la mort, après avoir été prégnant à la suite du diagnostic, ou des deuils successifs, s'est réduit avec le temps ou grâce à un cheminement de croissance personnelle et, chez certains, l'apparition des trithérapies semble être venue repousser encore davantage l'idée de la mort et son horizon. Ces médicaments ont permis à plusieurs hommes, souvent malgré un scepticisme initial, de retrouver une qualité de vie satisfaisante et une énergie vitale associée à la mise en place de projets professionnels, une réactivation des réseaux personnels et le retour à une vie interpersonnelle plus adéquate, même si les questions rattachées à la prévention sexuelle restent souvent problématiques (Lévy et al., 2003).

Parmi les femmes d'origine africaine (Bastien et al., 2003), la possibilité d'accéder à des services de santé compétents et à des traitements efficaces contre le VIH a permis de maintenir ou d'améliorer leur état de santé, leur permettant de mieux faire face aux défis complexes qu'elles ont à gérer : s'adapter, après leur immigration, aux nouvelles conditions de vie et à une nouvelle culture ; assumer de lourdes responsabilités familiales associées aux soins des enfants dont elles doivent s'occuper généralement seules et qui constituent pour elles une préoccupation essentielle. Dans cette perspective, les traitements leur fournissent une motivation supplémentaire pour demeurer en vie et contribuent à mobiliser leur énergie et à assurer l'atteinte de leurs objectifs de vie, bien que, comme dans le cas des hommes, la réorganisation de la vie amoureuse et sexuelle reste problématique.

Pour plusieurs de ces patients, hommes et femmes, l'adhésion au traitement semble ne pas poser de problèmes, les multithérapies étant considérées essentiellement comme des substances pharmacologiques, analogues à d'autres médicaments aux effets biomédicaux bien définis. Il s'agit alors de les absorber selon les consignes de la prescription, sans état d'âme particulier, le rapport au médicament étant essentiellement ramené au caractère technique du geste posé. Pour d'autres, par contre, le rapport aux médicaments est nettement plus problématique, donnant alors lieu à des perceptions plus ambivalentes, sinon hostiles.

\section{LE MÉDICAMENT :}

SOURCE D'AMBIVALENCE

Pour d'autres répondants, le médicament est perçu de façon plus ambivalente. On reconnaît certes sa valeur technique, mais la croyance en son efficacité reste chancelante, même si le traitement peut être fidèlement observé. 
Je continue la trithérapie, ça fait quatre ans, fidèlement, j'y crois et j'y crois pas, mais je les prends quand même (homme).

Quand je les prenais, je me disais quand même, il y a eu des progrès [...] On ne connaît pas les effets, mais je me disais au moins tant que ça ralentit la maladie et que ça allonge ta vie, j'ai choisi entre le moindre mal... D'abord tu les prends, on te dit ça guérit pas [...] Tu sais que ça guérit pas, je me dis au moins j'allonge mes jours de deux ou trois ans (femme).

[Les médicaments], c'est un répit, c'est la santé en capsules. En attendant que quelque chose de définitif arrive (homme).

Moi, je n'aime pas les médicaments, c'est ça mon problème [...] Je suis obligée de prendre les médicaments à tous les jours [...] Mais comme on n'a pas le choix, je vais les prendre sinon franchement (femme).

\section{LE MÉDICAMENT : SOURCE D'ANXIÉTÉ,} DE MALADIE ET DE MORT

Plusieurs participants interviewés, hommes et femmes, ressentent, à des degrés divers, des effets indésirables plus ou moins graves. Certains les envisagent comme un moindre mal comparativement au sida, s'y adaptant avec succès. D'autres, au contraire, confrontés à des difficultés d'adhésion au traitement, à des malaises ou à des maladies consécutives à la prise des antirétroviraux, les considèrent, même si le protocole thérapeutique se révèle objectivement efficace sur le plan de la charge virale, comme une source de détérioration de leur qualité de vie, en particulier, chez les hommes, lorsque le traitement a été commencé à titre préventif et avant que des symptômes particuliers se soient manifestés. La détérioration de l'apparence corporelle avec la lipodystrophie et / ou la lipoatrophie, les conséquences psychiques (dépression, perte de l'estime de soi, etc.) contribuent alors à des sentiments de précarité associés à une incertitude quant à l'avenir (durée de vie, qualité de la vie professionnelle, affective, sexuelle, etc.) qui remettent en question la contribution positive des médicaments au traitement du VIH / sida considéré à plus long terme.

C'est pas vrai que le monde vont bien à cause des médicaments, c'est pas vrai, c'est juste que ça a changé, avant ils mouraient. C'est pas mal plus difficile de vivre avec que c'était d'en mourir (homme).
Prendre des médicaments toute ma vie, seigneur, ça fatigue psychologiquement, physiquement, sur tous les plans. Je ne sais pas si les médecins réalisent ça quand ils nous prescrivent tous ces médicaments. On n'a pas le choix (femme).

Le médicament est alors considéré comme un moyen d'allonger l'espérance de vie, mais une vie tronquée et maladive que quelques répondants ne sont pas prêts à assumer à long terme :

Est-ce que ce n'était pas bien avant les médicaments? [...] Parce [qu'en ce moment] j'ai l'impression de vivre une agonie qui ne s'arrête pas. [...] Je vois les gens qui se rendent jusqu'au bord de la mort et qu'on ramène [...] et qu'on ramène encore. Je ne sais pas, si j'en arrive là, si ça va me tenter (homme).

Ces effets remettent en cause l'efficacité des traitements et soulignent leur portée iatrogène, toxique et antinaturelle sur l'organisme.

C'est sûr et certain, les médicaments doivent maganer le système pas à peu près. Je pense que lorsqu'on les prend, oui, ça nous aide, mais ça doit détruire d'autre affaires ça c'est certain. Ça doit être toxique à mourir ces affaires-là (femme).

Tout médicament est toxique, pour guérir, je pense que oui. Ça doit être toxique, peut-être pas nocif mais toxique (femme).

J'ai toujours vu dans les médicaments un truc nocif. Moi, je me dis qu'un organisme n'est pas fait pour les médicaments. Un organisme sain doit être sans médicament. C'est dans ma conception, ma petite logique. Donc, quand $[. .$.$] tu commences à avaler des$ médicaments ça te guérit un mal qui t'en cause un autre. C'est comme ça que je conçois les médicaments. Donc quand je peux éviter de prendre un médicament, je l'évite (femme).

De ce fait, le caractère bienfaisant de ce type de médication et l'espoir suscité par les nouvelles thérapies restent éphémères et temporaires.

C'est une idée aussi que [les médicaments], c'est pour avoir une meilleure qualité de vie. Mais la qualité de vie que j'ai, c'est que je me lève à onze heures. Je passe deux heures, l'aprèsmidi, couché. Je me couche de bonne heure, ce qui fait que ma journée je ne la vois quasiment pas. Ça, ce n'est pas une qualité de vie (homme).

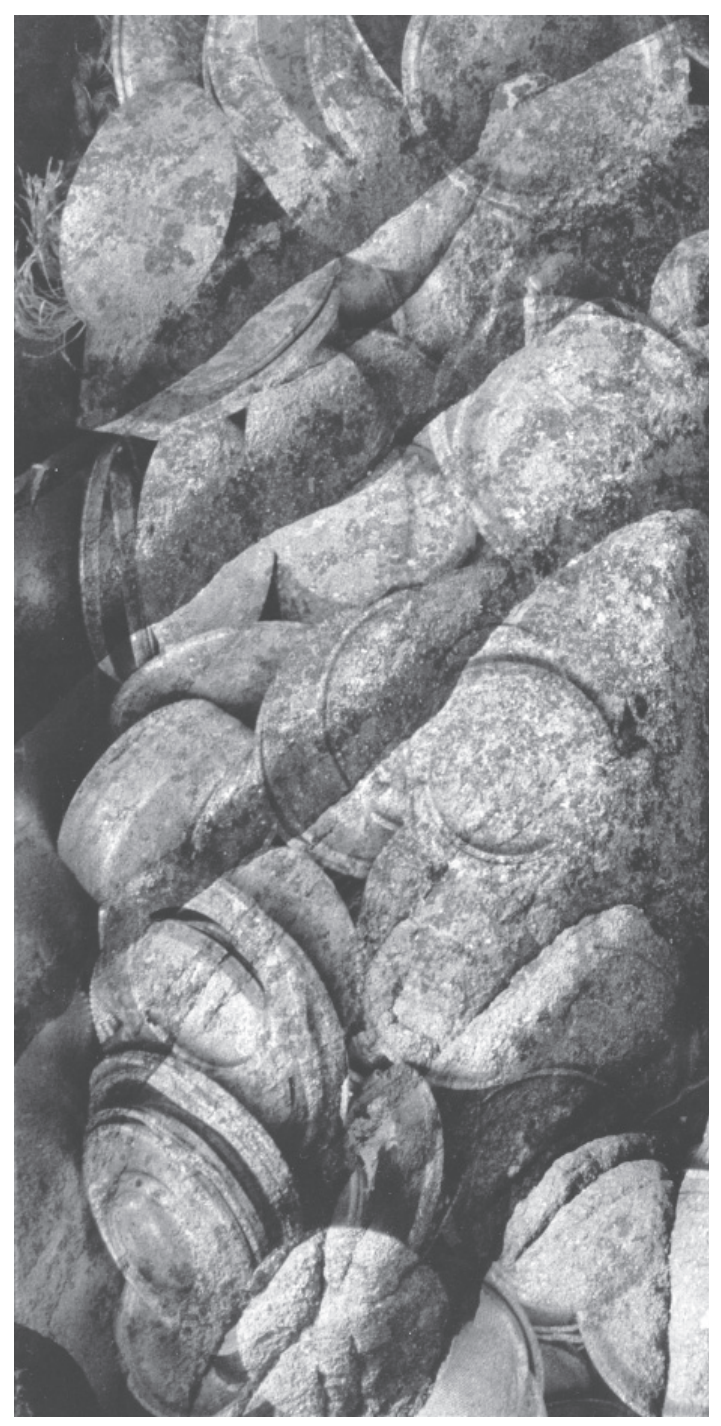

Lorsque les effets secondaires se chronicisent et s'accumulent et qu'une escalade médicamenteuse s'ensuit, le traitement apparaît alors souvent difficile à assumer. Le phénomène d'essoufflement thérapeutique vécu par les patients peut aller, dans certains cas, jusqu'à envisager d'arrêter le traitement :

Présentement je suis rendu à 18 médicaments par jour, ce qui fait que je me demande si c'est une bonne affaire tous ces médicaments. Des fois, j'aurais envie de tout ficher en l'air (homme).

Des effets secondaires, un par-dessus l'autre et des escalades de médicaments, de pilules pour contrer les effets secondaires. Je n'ai pas envie de vivre tout ça (homme).

Par jour, je prends 18 pilules, deux fois par jour. J'ai dit au docteur que c'était trop (femme). 


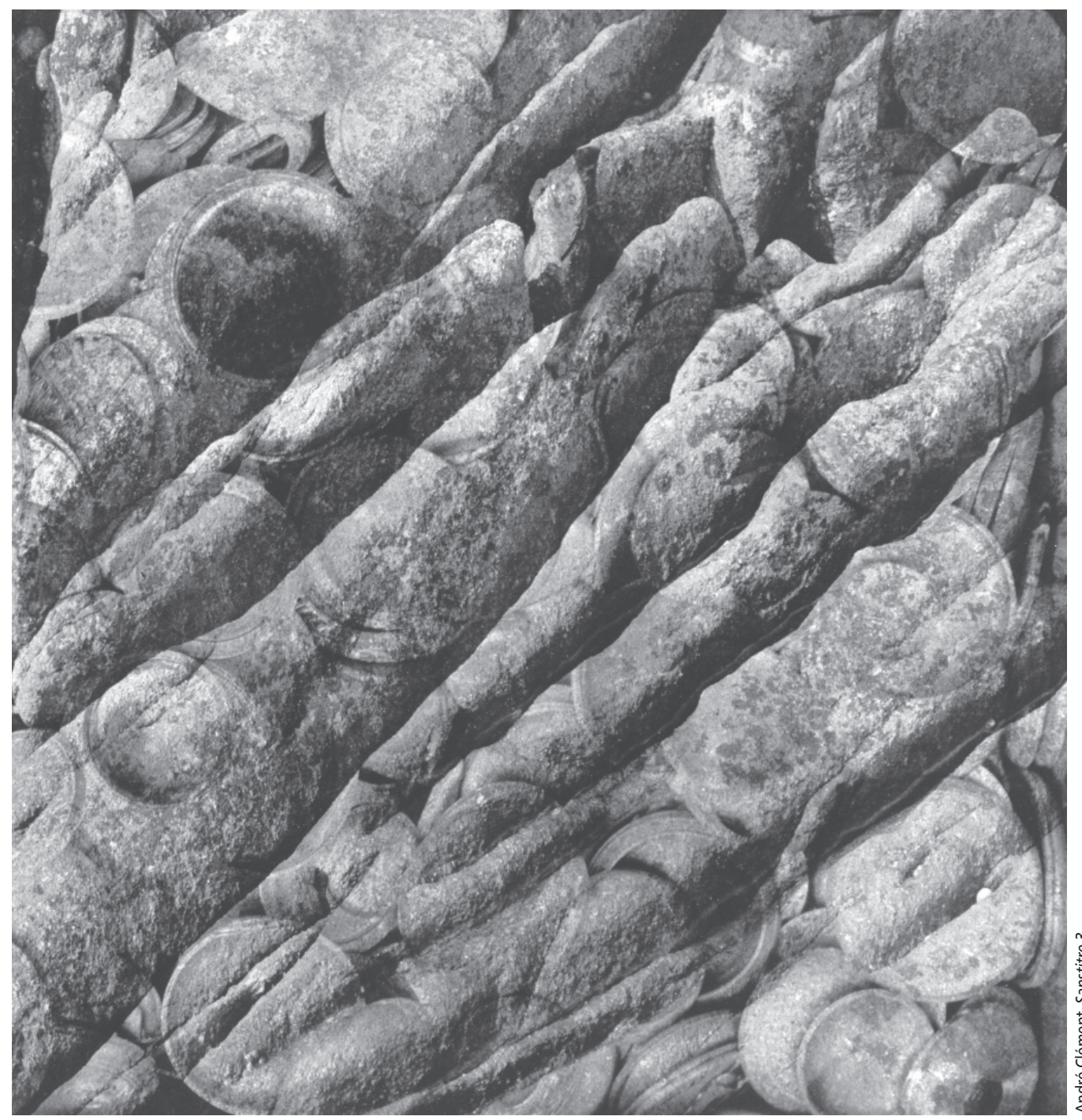

Dans certains cas, les PVVIH peuvent décider de prendre une vacance thérapeutique et de plus en plus de médecins les accompagnent vers des prises en charge comprenant de longues périodes d'arrêt du traitement, parallèlement à un suivi régulier de l'évolution de la maladie:

Quand j'ai décidé d'arrêter, je sentais que même mon corps était tanné, en fait j'étais tannée de prendre mes médicaments [...] je me disais, mon dieu, ça va me donner au moins le temps de vider tout ces toxiques de médicaments, prendre juste, juste un temps de répit, quoi, mais heureusement ça va bien. Donc même mon médecin est d'accord tout ça donc il y a pas de problème (femme).

\section{LE MÉDICAMENT :}

\section{UNE SUBSTANCE À APPRIVOISER}

On retrouve aussi chez les répondants des mécanismes d'apprivoisement des médicaments qui s'articulent sur des représentations variées du fonctionnement des alimentaires (jus de fruits ou de légumes), à la suite de la lecture d'ouvrages de naturopathes, de revues, de programmes de télévision, ou de conseils d'amis, apparaît comme une stratégie susceptible de contribuer à un plus grand bien-être physique et à améliorer l'immunité de l'organisme :

J'ai quand même l'impression que je m'intoxique un peu en prenant ces médicaments. De l'autre côté, pour contrebalancer ça, je vais faire des choses qui sont extrêmes dans l'autre sens : manger des légumes bio qui n'ont pas d'insecticides, des trucs super sains (homme).

Je commençais à comprendre comment le virus agit, comment l'immunité affecte, comment la maladie évolue et j'essaie de m'alimenter en conséquence. Des huiles naturelles, des fruits, tout ce qu'on dit que ça baisse l'immunité de la maladie. J'ai commencé à me prendre en charge. J'ai pris des régimes de jus naturels [...] $\mathrm{du}$ jus de betteraves [...] des prunes de Japon qui amènent du fer. [...] j'essaie tout ce qui amène des sels minéraux, les vitamines dont a besoin l'organisme. Les huiles de carottes, les huiles de betteraves (femme).

L'apparition des nouvelles thérapies antirétrovirales a profondément modifié la perception et les représentations de l'épidémie, participant à sa banalisation relative et à l'émergence d'un statut qui se rapproche de celui de maladie chronique. Cette tendance à la normalisation occulte cependant le fait que ces médicaments ne constituent pas une cure mais plutôt un moyen de contrôler la progression du virus et ses conséquences létales. Les lourdes contraintes de l'observance, qui jouent sur la temporalité de la vie quotidienne des patients (Broqua, 1999) et les effets bénéfiques ou indésirables contribuent à moduler les représentations et les perceptions des traitements parmi les PVVIH, comme le montrent les données qualitatives provenant de deux recherches menées à Montréal. Les représentations des médicaments, tout en se basant sur leur fondement biomédical les débordent pour inclure des dimensions symboliques et interprétatives complexes. Ainsi, elles sont l'objet de projections et investies d'une charge affective importante. Source d'espoir, de bien-être, de vie, le traitement est aussi perçu avec répulsion, car il est rappel constant de la maladie et de la mort et cause iatrogénique de nombreux maux qui contribuent à la détérioration de la qualité de vie. Le processus du deuil s'est ainsi vite transformé. Le malade doit apprendre à faire le deuil de sa qualité de vie. La mort demeure 
un enjeu, mais de façon indirecte, inéluctable et imprévisible. Les pertes reliées à leur qualité de vie finissent par miner l'espoir chez certains. L'isolement guette ces survivants nouveau genre.

Le médicament apparaît bien, comme le souligne Dagognet (1964, p. 27), «une matière équivoque et contradictoire, [...] toxique et bienfaisante en même temps, source de crainte et d'attirance, danger et secours, chose et esprit, forme apparente et force cachée ». Cette ambivalence rejoint les conclusions des recherches anthropologiques sur la construction et l'expérience des médicaments (van der Geest, Whyte et Hardon, 1996) qui montrent que les médicaments ne sont pas des substances techniques neutres mais qu'ils sont investis de significations et d'interprétations multiples. Celles-ci dépassent le strict référent biomédical (Benoist, 1999) et s'articulent aux différentes matrices socioculturelles et aux expériences personnelles variées qui en modulent les constructions et les perceptions. Ainsi les médicaments, selon van der Geest et al. (1996), font l'objet d'un engouement remarquable associé à leur efficacité et leurs effets positifs sur l'organisme, allant jusqu'à la guérison. À l'inverse, ils peuvent faire l'objet de critiques acerbes sinon d'un rejet à cause de leur toxicité, leur agressivité, leur artificialité et leurs effets iatrogéniques, et être associés à une forme de refus de l'emprise du système biomédical. Ces contradictions se retrouvent dans le cas des thérapies antirétrovirales qui marquent cependant une nouvelle étape dans l'évolution de l'épidémie qu'elles contribuent à transformer.

\section{Bibliographie}

BASTIEN, R., M. FERNET, J.J LÉVY, J. OTIS, J. SAMSON, M. HARERIMANA, M. RATEAU, S. RICHARD, I. GOUPIL, G. TROTTIER, R. PELLETIER, N. LAPOINTE et M. BOUCHER, "Vivre avec les médicaments antirétroviraux et l'infection au VIH/ sida: l'univers amoureux et sexuel de femmes montréalaises d'origine africaine sous traitements », dans J.J. Lévy, J. Pierret et G. Trottier (dir. publ.), Les traitements antirétroviraux: expériences et défis (sous presse).
BENOIST, J. (1999). «À propos du rapport entre dimension technique et médiation symbolique dans le médicament », dans O. Faure (dir. publ.), Les thérapeutiques: savoirs et usages. Lyon, Collection Fondation Marcel Mérieux, p. 383-394.

BRASHERS, D.E., J.L. NEIDIG, L.W. CARDILLO, L.K. DOBBS, J.A. RUSSELL et S.M. HAAS (1999). "In an important way, I did die: uncertainty and revival in persons living with HIV or AIDS », AIDS Care, vol. 11, $\mathrm{n}^{\circ}$ 2, 201-219.

BROQUA, C. (1999). "Résonances individuelles et sociales des nouveaux traitements de l'infection au VIH», dans O. Faure (dir. publ.), Les thérapeutiques: savoirs et usages. Lyon, Collection Fondation Marcel Mérieux.

CHAST, F. (1995). Histoire contemporaine des médicaments. Paris, La Découverte.

DAGOGNET, F. (1994). La raison et les remèdes. Paris, Presses universitaires de France.

DE MONTIGNY, J. (1997). "Aspects thanatologiques liés au sida", dans J.J. Lévy et H. Cohen (dir. publ.), Le sida, aspects psychosociaux, culturels et éthiques, Montréal, Éditions du Méridien, p. 413-433.

DELFRAISSY, J.-F. (1999). Prise en charge thérapeutique des personnes infectées par le VIH. Paris, Flammarion.

DETELS, R., A. MUNOZ, G. MCFARLANE, L.A. KINGSLEY, J.B. MARGOLICK, J. GIORGI, L.K. SCHRAGER et J.P. PHAIR (1998). "Effectiveness of potent antiretroviral therapy on time to AIDS and death in men with known HIV infection duration ", Journal of American Medical Association, vol. 280, p. 1497-1503.

FERNET, R. (1999). «Démarches de la pensée suicidaire et impacts dans la personne", dans M.L. Morin (dir), Le suicide chez les jeunes. Un cri pour la vie, Montréal, Médiaspaul, p. 83-122.

LÉVY, J.J. et J. OTIS (2000). « Le sida comme événement », dans G. Soussana et J.J. Lévy (dir. publ.), Actualités de l'événement. Montréal, Éditions Liber, p. 181-195.

LÉVY, J.J., R. BASTIEN, A. BOURDAGES, J. OTIS, R. PELLETIER et G. TROTTIER (2001). Les représentations sociales des nouvelles thérapies contre le VIH-sida et leurs répercussions dans la vie d'hommes ayant des relations sexuelles avec d'autres hommes et vivant avec le VIH. Rapport de recherche, Département de sexologie, Université du Québec à Montréal.
NOUSS, A. (1997). "La culture du sida: discours critiques et approches esthétiques", dans J.J. Lévy et H. Cohen (dir. publ.), Le sida, aspects psychosociaux, culturels et éthiques, Montréal, Éditions du Méridien, p. 485-508.

SANTÉ CANADA (2003). Communiqué VIH / sida. vol. 2 , no 1 . Hiver. www.hc-sc.gc.ca/hppb/ vih-sida/strat-can/communiques-jan.html

SÉGUIN, M. et L. FRÉCHETTE (1995). Le deuil, une souffrance à comprendre pour mieux intervenir. Montréal, Logiques.

SETBON, M. (2000). "La normalisation paradoxale du sida». Revue française de sociologie, vol. $41, \mathrm{n}^{\circ} 1$, p. 61-78.

TROTTIER, G., J.J. LÉVY, J. OTIS, R. BASTIEN, R. PELLETIER, A. BOURDAGES et M. FERNET (2003). «Effets des nouvelles thérapies sur la qualité de vie des HARSAH vivant avec le VIH/sida», dans J.J. Lévy, D. Maisonneuve, H. Bilodeau et C. Garnier (dir. publ.), Enjeux psychosociaux de la santé, Sainte Foy, Presses de l'Université du Québec, collection Santé et Société, p. 301-313.

VAN DER GEEST, S., S.R. WHYTE et A. HARDON (1996). "The anthropology of pharmaceuticals : A biographical approach ", Annual Review of Anthropology, vol. 25, p. 153-178.

\section{Notes}

1.Étudiante de maîtrise, Faculté d'anthropologie, Université de Lyon II Lumière.

2. Professeur(e), Département de sexologie, Université du Québec à Montréal.

3. Chercheur, Unité Écologie humaine et sociale, Direction de la santé publique de MontréalCentre.

4. Professeur associé, Faculté de théologie, d'éthique et de philosophie, Université de Sherbrooke.

5. Professeur associé, École de service social, Université Laval.

6. Coordonnatrice de recherche, Centre maternel et infantile sur le sida, Hôpital Sainte-Justine.

7. Médecin, Centre maternel et infantile sur le sida, Hôpital Sainte-Justine.

8. Présidente du C.A., Groupe d'action pour la prévention de la transmission du VIH et l'éradication du Sida (GAP-VIES).

9. Directrice générale, Centre de ressources et d'interventions en santé et sexualité (CRISS).

10. Agent de recherche, Département de sexologie, Université du Québec à Montréal. 